\title{
EDITORIAL
}

\section{Evidence-based Medicine: An Introspection!}

Vandana M Gudhe

How to cite this article: Gudhe VM. Evidence-based Medicine: An Introspection! Int J Recent Surg Med Sci 2018;4(1):1.

\section{Source of support: Nil}

\section{Conflict of interest: None}

Research forms a strong basis in our education system, which has shown a significant improvement all these years. The dissemination of research findings and use of clinical evidence benefit the populations and health care systems and influence public health issues globally, offering public and patient education, thus contributing to a quality health care on a global scale. It also offers a platform to address issues related to clinical decision making. It basically aims at improving the insight and knowledge of the clinician.

Evidence-based medicine is a specific approach in the practice of medicine to improve clinical decision making of the individual physicians across the world to ensure better clinical outcomes. We are here on an agenda to improve, develop, disseminate, and implement research evidence for a better health outcome.

\section{Assistant Professor}

School of Epidemiology and Public Health, Datta Meghe Institute of Medical Sciences University, Wardha, Maharashtra, India

Corresponding Author: Vandana M Gudhe, Assistant Professor, School of Epidemiology and Public Health, Datta Meghe Institute of Medical Sciences University, Wardha Maharashtra, India, e-mail: vclohana@gmail.com
Research should be driven with the aim of addressing a knowledge gap and generating evidences to add to the existing literature. In the present era, research projects and scientific publications have been encouraged in academics and have been moreover made mandatory. This perhaps has increased the proportion of doctors and clinicians getting involved in research, but perhaps has also become a reason for perpetuating research only for the purpose of mere publications, promotions, and appraisal.

Thus, the evidence generated through research does not get translated into actual practice; or the evidence generated has been of a poor quality due to faulty methodology that is unable to prove the study hypothesis or get a definitive answer to the research question. These are the hindrances leading to poor quality research, where the evidence generated is partly withheld (bias), i.e., piecemeal dissemination, violation of ethical considerations, etc. posing a false picture. This has definitely caused a breach in scientific communication, decreasing the quality of research. We need to devise strategies that work to rule out these fallacies and encourage better research practices.

As a clinician and researcher, we encourage scientific writing and authenticity in research methods generating high quality evidence. This will ensure future sustenance and development of imperative research.

We also encourage more articles on the controversial health topics, that will have implications for the patients, health planners and policy makers. 